\title{
Effect of the Volume of Magneto-rheological Fluid on Shear Performance
}

\author{
X.h Liu, L Jin \\ School of Mechanical Engineering, Shanghai institute of Technology, Caobao Road, China, Liu_xuhui79@163.com
}

\begin{abstract}
As a kind of smart material, MR (magneto-rheological) fluid is dramatically influenced by the external magnetic field and can change from the liquid state to semi-solid state in several milliseconds. In this paper, the effect of different volume of MRF on its shear performance is proposed. A set of testing systems, including the plate-on-plate MRF shearing test rig, is built up to measure the relationship between the produced shear torque and the added volume of MRF in different current. The variation of magnetic flux density in the shear gap is measured by teslameter and simulated before and after MRF is added. The results validate the effect of volume on the shear torque experimentally.
\end{abstract}

Keywords: Shear performance, MR fluid, the volume, magnetic field

\section{INTRODUCTION}

$\mathrm{M}$ AGNETO-RHEOLOGICAL fluids are stable disperse systems composed of two phases, such as magnetic particles (of size 3-10 $\mu \mathrm{m}$ ) and hydrocarbon. The fluids can change from a liquid to a near-solid state in the presence of a magnetic field in milliseconds [1]-[2], which makes them widely applied on the vehicles, engineering structures and other fields.

As to the typical shear MRF dampers, the working cylinder is full of MR fluid [3]-[5], during the working process of dampers, the produced leakage will be unavoidable and will shorten the life of the damper, especially to the disk-disk shear dampers, as well as weaken the MR effect (mainly in shear performance).

However, the previous studies focused on the measurement of the characteristics or new designs of the MRF dampers [3], the influence of volume on the shear performance has been seldom investigated. Besides, our early works have mentioned the effect of volume on the characteristics of MR [6]. Many researchers [7]-[8] have studied the variation of the mechanical property (or the physical parameters) of viscoelastic fluids (including MR or magnetic fluid), and they found that, considering a large quantity of the existing gas bubble, the property will be influenced dramatically, that is, the volume of MR fluid has a visible effect on the shear performance (or the other physical parameters).

The paper presents the factors on the mechanical property of MR fluid in rotary shearing mode based on the Bingham model, and the plate-on-plate MRF shearing test rig is built up to investigate the relationship between the added volume of MR fluid and its shear performance. The magnetic field (mainly magnetic flux density) in the shear gap is measured by teslameter and simulated by FEM software before and after MR fluid is added into the shear gap, and then, the effect of different volume of MR fluid on the shear performance is investigated in different current experimentally.

\section{THEORY AND PARAMETERS OF MEASUREMENT}

Here, the working principle of MR fluid devices is the rotating shear mode, as shown in Fig.1. MR fluid is put into the shear gap between the two shear disks, it is concentrated toward the center of the plates, whether the volume of MR fluid is low or high, and the rotating radius is denoted $r$ and the gap size $h$. From Fig.1, the relationship between the shearing rate and the angular velocity can be derived from the strain of MRF in shearing state.

$$
\text { Shear disk MR fluid }
$$

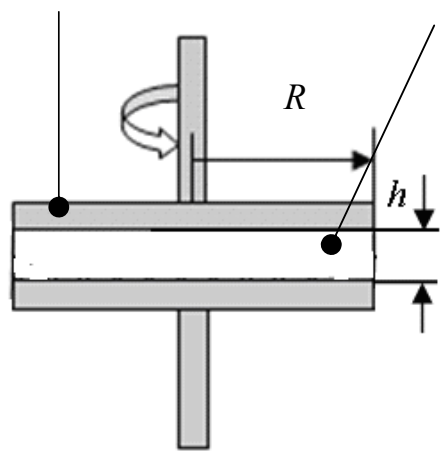

Fig.1. The working mode of MR fluid

Here, the shear strain

$$
\gamma=\frac{x}{h}
$$

Here, $x$ is the linear displacement, thus, the shearing rate can be expressed

$$
\dot{\gamma}=\frac{\dot{x}}{h}
$$

Where, $\dot{\gamma}$ is the shearing rate, $\dot{x}$ is the linear speed. This is converted to the angular velocity 


$$
\dot{\gamma}=\frac{\omega r}{h}
$$

Here, $r$ is the arbitrary radius of the shearing discs and $\omega$ is the angular velocity.

Beside the shearing rate, in the action of magnetic field, the resisting torque (or the shear torque) generated by the shear movement is also related to the yield stress of MRF, therefore, the shear torque should be calculated according to the yield stress. It can be deduced from Fig.2. This is the top view of the MRF in the two shear parallel-plate fixture.

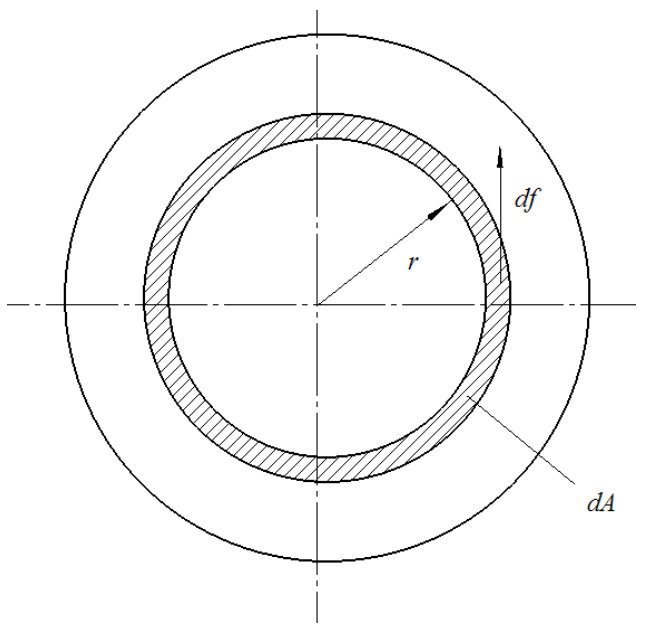

Fig.2. The calculation sketch of shear torque of MRF

In Fig.2, the area of MRF in action is $d A$, the force applied on MRF is $d f$, thus, the shear stress, $\tau$, is as (4)

$$
\tau=\frac{d f}{d A}
$$

As the non-Newton characteristics of MRF in the action of magnetic field, the mechanics model can be assumed as the Bingham model, that is,

$$
\tau=\tau_{y}+\eta \dot{\gamma}
$$

Here, $\tau_{y}$ is the shear yield stress of MRF, which is related to the characteristics of MRF, combined with (4) and (5)

$$
d f=\left(\tau_{y}-\eta \dot{\gamma}\right) d A
$$

The area of the toroidal shape can be approximated as

$$
d A=2 \pi r d r
$$

(7) can be substituted into (6), which yields

$$
d f=\left(\tau_{y}-\eta \dot{\gamma}\right) \cdot 2 \pi r d r
$$

According to the definition of the shear torque, the following equation can be obtained

$$
d T=r \times d f=r\left(\tau_{y}-\eta \dot{\gamma}\right) \cdot 2 \pi r d r
$$

(3) and (8) are substituted into the (9); (9) can be expressed

$$
d T=2 \pi\left(r^{2} \tau_{y}-r^{3} \varpi \eta / h\right) d r
$$

The integration of (10)

$$
\int d T=\int_{0}^{R} 2 \pi\left(r^{2} \tau_{y}+\frac{\varpi \eta r^{3}}{h}\right) d r
$$

That is,

$$
T=2 \pi\left(\frac{R^{2} \tau_{y}}{3}+\frac{\varpi \eta R^{4}}{h}\right)
$$

Equ.12 gives the relationship between the shear torque and the yield stress of MRF, as to the yield stress, in the condition of the gap it is filled with MRF, which is related to the magnetic field in the gap. Besides, from Equ.12, if the type of MR fluid is given, and the parameters of devices, including the radius $R$ and gap size $h$, the angular velocity $\omega$ are constant, the maximum torque is related to the shear yield stress. Here, the shear yield stress $\tau_{y}$ is mainly decided by the magnetic field, (it is maybe affected by the shearing rate, but it is small), thus, once the device is built up, the maximum torque will be related to the characteristics of MR fluid and magnetic field. These relevant theories and experiments have been studied before [1], but if the shear gap is not full of MRF, what will happen to the shear torque of MRF?

\section{Methods of MEASUREMENT}

A plate-on-plate MRF shearing test rig was developed to measure the shear torque of MRF with different volume. Fig. 3 is the design sketch of this test rig.

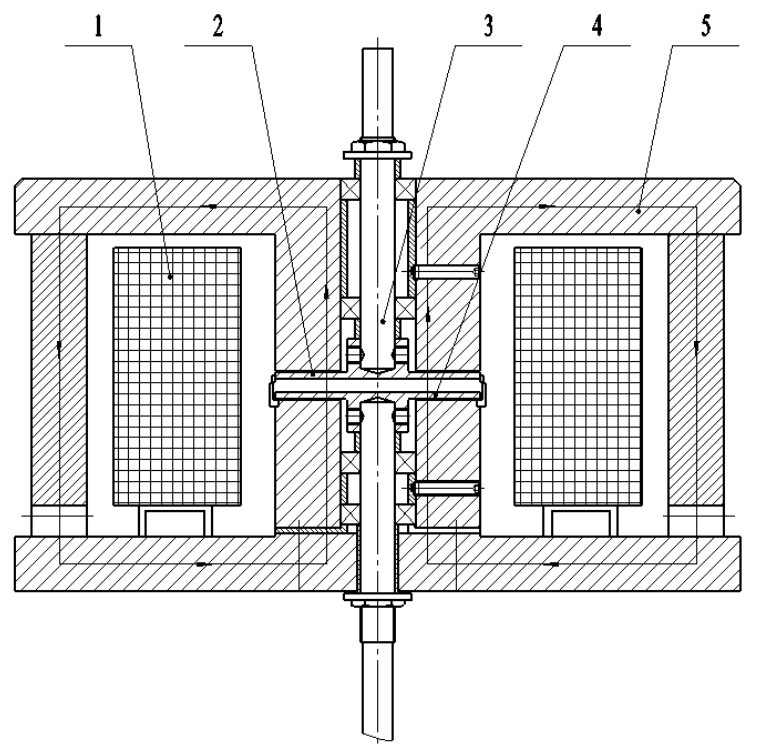

Fig.3. Sketch of disk-type MRF test cell. 1: Coil, 2 and 4: Parallel shear disks, 3 and 5: Highly permeable materials. 
The test specimen MR fluid is MRF-132AD of Lord Corp., which consists mainly of 3 components including magnetisable particles in micron-sized, hydrocarbon based oil and other additives, e.g., suspending agent, thixotropes, anti-wear and anti-corrosion additives, and friction modifiers. It is a hydrocarbon-based suspension of around $30 \%$ (volume) iron particles. Its density is $3.06 \mathrm{~g} / \mathrm{cm}^{3}$. The size and shape distributions of the magnetisable particles were examined by scanning electron microscopy (SEM). The MR particle shape was found to be nearly spherical and typical dimensions around $1-5 \mu \mathrm{m}$ in diameter.

The relationship of magnetic field density B to current I was simulated by FEM. Fig.4(a) is the design sketch used for the simulation, Fig.4(b) shows the distribution of magnetic density flux with no MRF in the gap when the current is $0.65 \mathrm{~A}$. Fig.5 illustrates the comparison of the simulation by FEM software and the experimental measurement by teslameter for the magnetic density flux in different current.

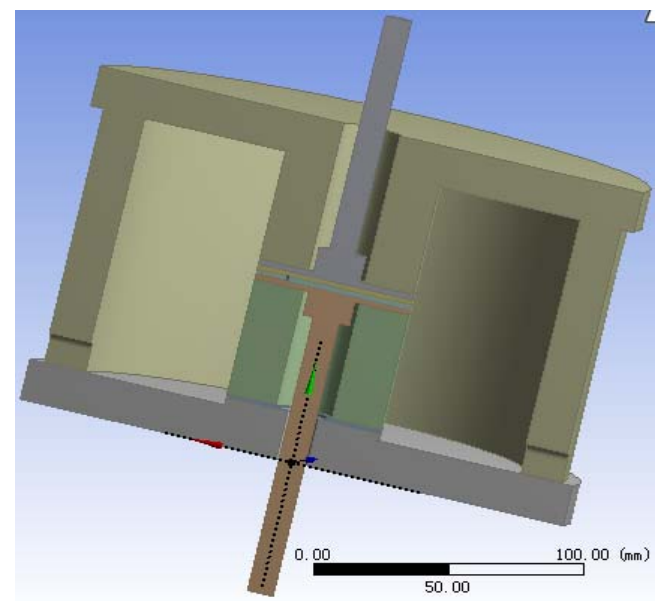

a) Simulation design

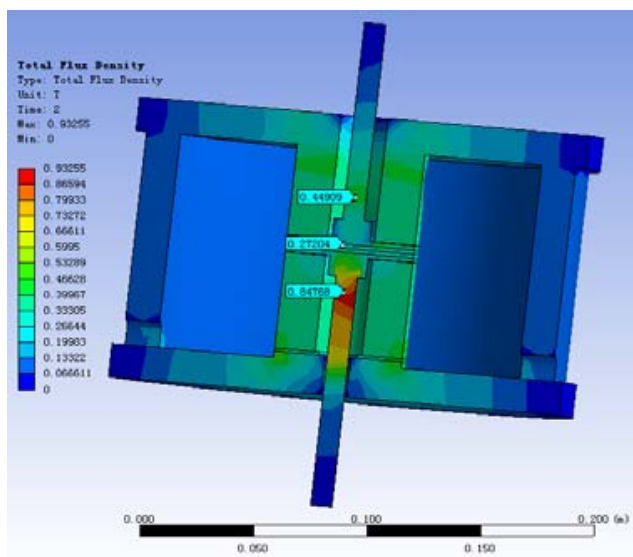

b) Simulation result

Fig.4. The distribution of magnetic flux density (Current $=0.65 \mathrm{~A}$, and gap size $h=1.00 \mathrm{~mm}$ )

As the magnetic flux density is the function of the added materials in the gap, and different when the gap is full of MRF and is without MRF, the measurement is necessary to value the difference.
Fig. 5 shows the variation in the following conditions, that is, the simulation and the measurement when no MRF is in the shear gap, the other is the measurement when the gap is full of MRF. It is obvious that because of permeability differences, the magnetic permeability of MRF is larger than air, the magnetic field $B$ in the shear gap full of MRF is higher than that of the other case.

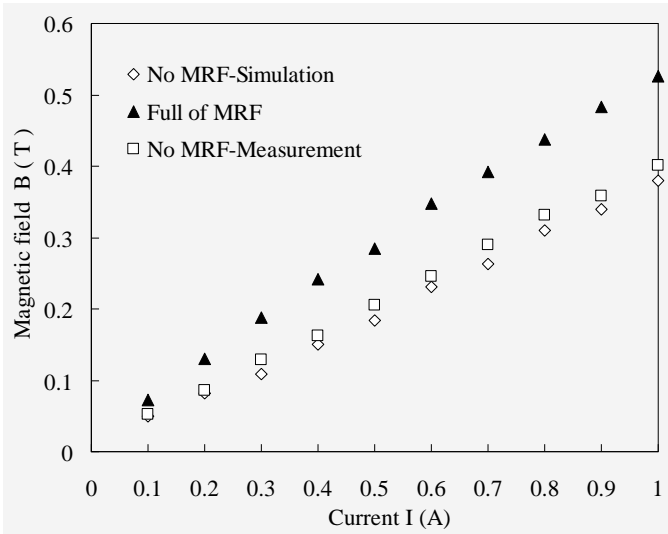

Fig.5. Magnetic field vs. Current

\section{RESUlTS AND DISCUSSION}

A certain amount of MRF is added into the shear gap between the two rotary disks by the injector, the shear torque measured will change with the different volume and the current. The following Fig.6 shows the relationship between the shear torque of MRF and the current in the different added volume.

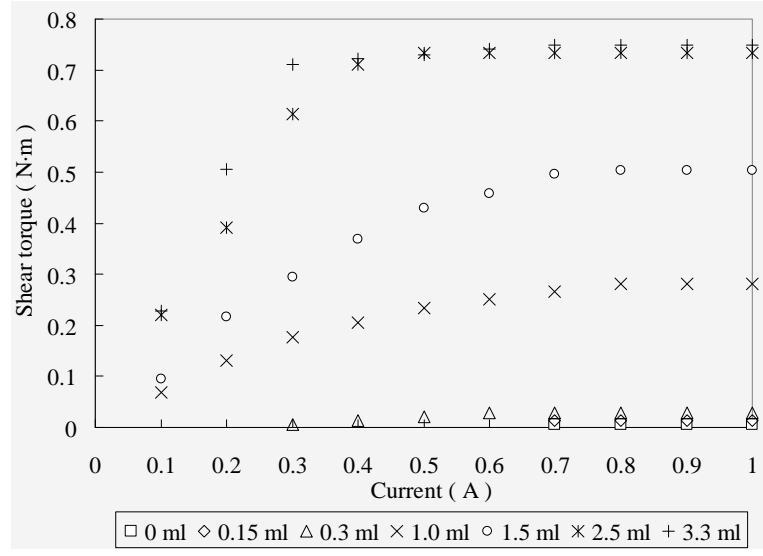

Fig.6. Shear torque vs. Current of different volume of MRF (Gap size is $1.0 \mathrm{~mm}$ )

In order to indicate the difference of the different volume clearly, the current is adjusted to $0.5 \mathrm{~A}$, Fig.7 gives the relationship between the shear torque of MRF and the added volume.

From Fig.6, we can conclude that,

1) When the same volume of MRF is added into the shear gap, with the current increased, if the current is less than 0.6 $\mathrm{A}$, the shear torque will increase clearly. Once the current exceeds $0.6 \mathrm{~A}$, the incremental will keep stable with the current continuing to increase, that is, when the current is 
0.6 A, the shear stress of MRF produced by magnetic field will arrive at its maximum and will become saturated.

2) If MRF with the volume of $3.3 \mathrm{ml}$ is added into the shear gap, by numerical calculation, we find that the shear gap will be filled with MRF, here, if the current exceeds 0.3 $\mathrm{A}$, the shear torque of MRF will be stable and not increased, and will arrive at the maximum, which shows, that if the shear gap is not full of MRF, the larger current will be required for the purpose of the maximum shear stress of MRF produced by the magnetic field.

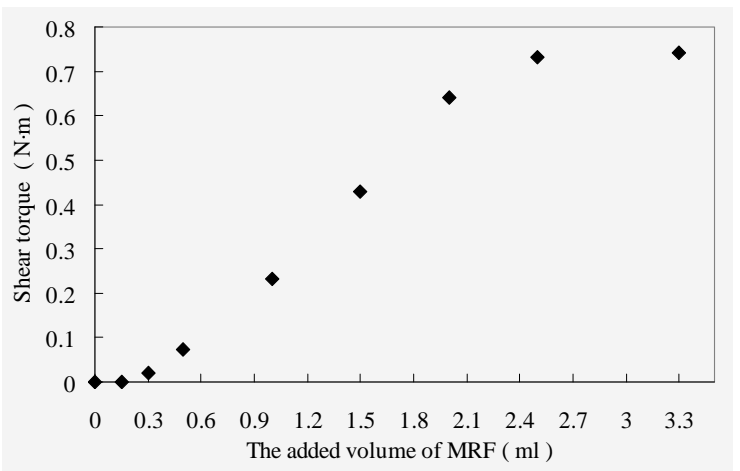

Fig.7. Shear torque and the volume

Fig.7 shows that with the added volume of MRF increased, the shear torque will increase clearly, and with the shear gap to be filled with MRF, the shear torque will approach stability.

In Fig. 6 and 7, as the friction torque and precision of the test rig exists, only the added volume of MRF exceeds 0.15 $\mathrm{ml}$, the shear torque will be detected, which will provide help for the engineering application.

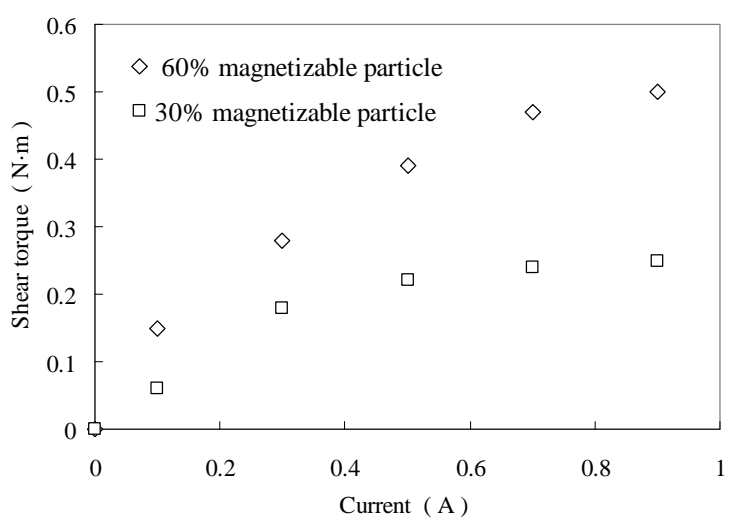

Fig.8. Shear torque vs. Current of different volume percentage of magnetisable particles

(Gap size $1.0 \mathrm{~mm}$, volume $1.0 \mathrm{ml}$ )

Moreover, the content of MR fluid's magnetisable particles represents the volume of MR fluid; therefore, the shear torque will change with the different volume percentage of magnetisable particles in MR fluids, as to the different volume percentage of magnetisable particles (with $30 \%$ and $60 \%$, MRF-132AD), the shear torque is compared experimentally on the following conditions: the gap size is $1.0 \mathrm{~mm}$, the current $0.4 \mathrm{~A}$ and the volume $1 \mathrm{ml}$, the results are shown in Fig.8.
Fig.8 shows that, if given the test rig (or device) and the volume of MRF in the same current, the shear torque with $60 \%$ magnetisable particles is larger than $30 \%$, and with the current increased, the shear torque will increase; when the current arrives at about $1.0 \mathrm{~A}$, the shear torque with $30 \%$ magnetic particles will get to the maximum, which is an advance to $60 \%$.

\section{CONCLUSION}

The relationship between the shear torque of MRF and the added volume is investigated experimentally. The factors on the shear torque are derived by the rotary shear mode, and then, the magnetic flux density in the shear gap is simulated by FEM software and measured before and after the shear gap is filled with MRF. The experimental results indicate that in the initial state of the increased current, the shear torque will increase clearly. Once the current exceeds the critical value, the shear stress of MRF will become saturated. Furthermore, the investigations also show that with the added volume of MRF increased, the shear torque will increase clearly. If the shear gap is filled with MRF, the shear torque will approach to stability. The paper also validates that the shear torque produced by MRF is sensitive to the volume of MRF experimentally.

\section{ACKNOWLEDGMENT}

The authors would like to thank Dr. Patrick Wong of City University of Hong Kong and Dr. Wen Wang of Shanghai University for their valuable help and advice.

\section{REFERENCES}

[1] Jolly, M.R., Bender, J.W., Carlson, J.D. (1999). Properties and applications of commercial magnetorheological fluids. J. Intell. Mater. Syst. Struct., 10 (1), 5-13.

[2] Ashour, O., Rogers, C.A., Kordonsky, W. (1996). Magnetorheological fluids: Materials, characterization, and devices. J. Intell. Mater. Syst. Struct. 7 (2), 123-130.

[3] Koo, J.H., Goncalves, F.D., Ahmadian, M. (2006). A comprehensive analysis of the response time of MR dampers. Smart Mater. Struct., 15, 351-358.

[4] Duan, Y.F., Ni, Y.Q., Ko, J.M. (2006). Cable vibration control using magnetorheological (MR) dampers. $J$. Intell. Mater. Syst. Struct., 17 (4), 321-325.

[5] Carlson, J.D. (2005). MR fluids and devices in the real world. Int. J. Mod. Phys. B., 19 (7-9), 1463-1470.

[6] Liu, X.H., Wong, P.L., Wang, W., Liu, K.P. (2009). Modelling of the B-field effect on the free surface of magneto-rheological fluids. J. Phys. Conf. Ser., 149, 012072.

[7] Shimaa, A., Tsujinoa, T., Nanjoa, H. (1986). Nonlinear oscillations of gas bubbles in viscoelastic fluids. Ultrasonics, 24 (3), 142-147.

[8] Huang, G., Hong, R., Li, H., Ding, J. (2008). Experimental and numerical investigations of rising gas bubble with different initial shape in a magnetic fluid. Comput. Appl. Chem., 25 (6), 141-144.

Received August 9, 2010. Accepted May 3, 2011. 